\title{
Aggression and Serotonin
}

\section{-Contribution of Inhibitory Mechanisms-}

\author{
Shuichi Ueda', Atsuko Ishizuya-Oka1, Akira Nishimura², Yoshihiro Takeuchi ${ }^{2}$ \\ and Kanji Yoshimoto ${ }^{3}$

\begin{abstract}
${ }^{1}$ Department of Histology and Neurobiology, Dokkyo University School of Medicine, Mibu, Tochigi 321-0293 and ${ }^{2}$ Department of Pediatrics, and ${ }^{3}$ Department of Legal Medicine, Kyoto Prefectural University of
\end{abstract} \\ Medicine, Kawaramachi-Hirokoji, Kamigyo-ku, Kyoto 602-0841
}

Received for publication December 8, 1998

\begin{abstract}
Transplantation of fetal serotonergic neurons into the hypothalamus restored muricide (mouse killing behavior) in the rat with raphe lesion induced by 5,7-dihydroxytryptamine (selective serotonergic neurotoxine). Immunohistochemical and neurochemical studies indicated that recovery of serotonergic innervation in the lateral hypothalamic area
\end{abstract}

by the graft brought about the inhibition of muricide. The grafted serotonergic neurons are strongly related to the inhibitory action on the muricide. Transplanted serotonergic neuron system can result in a reinnervation of the host, and leads to a reestablishment of the aggressive behavior.

Key words: Aggressive behavior, Rat, Transplantation, Serotonin, Immunohistochemistry

\section{Introduction}

Aggressive behavior is an instinctive and essential behavior in many species [7]. Especially, attacks on other species of animals are based on predatory behavior in wild animals $[1,4,7,10]$. Studies of the neuronal mechanisms of this aggression may lead researchers to important clues in understanding the domestication of wild animals.

Mouse-killing behavior (muricide) in the rat is one of the aggressive behaviors $[1,8,12]$. Because the killed mice are frequently eaten, muricide is considered predatory aggression. An increasing body of evidence suggests that serotonin may play a central role in the regulation of muricide $[2,3,6,11,13,16]$. However, it is not clearly understood which particular structures receiving serotonergic innervation might be involved in the control of muricide.

Correspondence to: Dr. Shuichi Ueda, Department of Histology and Neurobiology, Dokkyo University School of Medicine, Mibe, Tochigi 321-0293, Japan.

\section{Materials and Methods}

Experiment 1. (Exp. 1)

The first step in the present study investigated which part of the serotonergic projection involved muricide control. We injected serotonergic neurotoxin (5,7dihydroxytryptamine, 5,7-DHT) with catecholaminergic uptake blocker desipramine into several brain regions (Fig. 1). Adult Wistar male rats (weighing $300 \mathrm{~g}$ ) received desipramine followed by bilateral stereotaxic application of 5,7-DHT ( $5 \mu \mathrm{g}$ in $1 \mu \mathrm{l}$ of ascorbic acid and saline) into the olfactory bulb $(n=20)$, septum $(n=20)$, amygdala $(n=20)$, lateral hypothalamic area (LHA, $n=20$ ), ventromedial hypothalamus $(n=20)$, dorsal hypothalamic area $(n=20)$ and dorsal and medial raphe nuclei $(\mathrm{DRN}+\mathrm{MRN}, \mathrm{n}=35)$. Control rats $(\mathrm{n}=20)$ received a sham injection of the ascorbic acid and saline into the DRN + MRN. One week later, the incidence and mean latency of the mouse-killing response to mice within 15 min were recorded. 


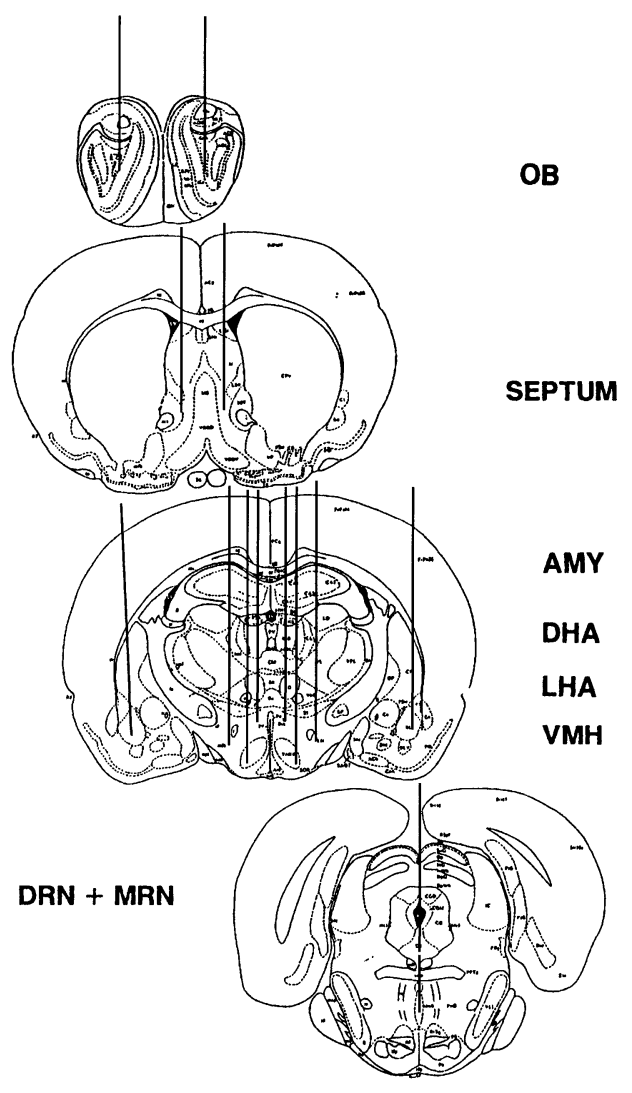

Fig. 1. Schematic showings of coronal sections through the brain depicting the 5,7-DHT injection sites. OB, olfactory bulb; SEPTUM, lateral septal nucleus; AMY, basolateral amygdaloid nucleus; DHA, dorsal hypothalamic area, LHA, lateral hypothalamic area; VHM, ventromedial hypothalamic nucleus; DRN, dorsal raphe nucleus; MRN, median raphe nucleus.

\section{Experiment 2. (Exp. 2)}

A gender difference in aggressive behavior has been reported. To clarify the sex steroid effects on mouse-killing behavior, adult female Wistar rats and male Wistar rats 2 weeks after castration and implantation with an osmotic mini-pump filled with estrogen (E2) received injection of 5,7-DHT into DRN+MRN.

\section{Experiment 3. (Exp. 3)}

In the third step of this study, we examined whether locally transplanted fetal serotonergic tissue could exert functional regulation over this complex, lesion-induced emotional behavior in adult rats. Adult Wistar male rats (weighing $300 \mathrm{~g}$ ) received desipramine followed by stereotaxic injection of 5,7-DHT into DRN + MRN $(n=25)$. One week later, mouse-killing response was checked, and then fetal raphe or cerebellum (as a control) tissues were stereotaxically placed bilaterally into the area just dorsal to the LHA of muricide rats. After 7 weeks of behavioral recording, the brains of the transplanted rats were analyzed for monoamine contents using HPLC with electrochemical detection (12 of raphe and 5 of cerebellum transplant group) and the immunohistochemistry was examined using serotonin antiserum (4 of each transplant group).

\section{Involvement of Serotonergic Mechanisms on Muricide}

In Exp. 1, an injection of LHA significantly increased the muricide incidence $(75 \%)$ similar to that in the $\mathrm{DRN}+\mathrm{MRN}$ injection group (80\%), whereas injections in other areas proved ineffective (Table 1.).

There was no difference in muricide incidence between normal males and the groups in Exp. 2.

In Exp. 3, the transplantation of fetal raphe tissue into 5,7-DHT treated rats resulted in the prolonged latency of mouse killing until 3 weeks after transplantation. After 4 weeks of raphe transplantation, muricide was restored (Fig. 2). Immunohistochemical and neurochemical analyses demonstrated the recovery of serotonergic innervation in the LHA by the graft inhibited muricide. The grafted serotonergic neurons were strongly related to the inhibition of muricide (Figs. 3, 4). Transplanted serotonergic neuron system can result in a reinnervation of the host, and lead to reestablishment of the aggressive behavior.

Table 1. Induction of muricide in rats 7 days after 5,7-DHT injection. The results were analyzed by mean of Fisher's exact probability test.

\begin{tabular}{lccc}
\hline injection site & No. of muricide rats/No. of animals tested & $\mathrm{P}^{*}$ & $\mathrm{P}^{+}$ \\
\hline DRN+MRN (saline) & $2 / 20$ & & $<0.0001$ \\
DRN+MRN & $28 / 35$ & $<0.0001$ & \\
OB & $4 / 20$ & $\mathrm{~ns}$ & $<0.05$ \\
SEPTUM & $4 / 20$ & $\mathrm{~ns}$ & $<0.05$ \\
AMY & $4 / 20$ & $\mathrm{~ns}$ & $<0.05$ \\
LHA & $26 / 35$ & $<0.0001$ & $\mathrm{~ns}$ \\
VMH & $8 / 16$ & $<0.05$ & $<0.05$ \\
DHA & $4 / 10$ & $\mathrm{~ns}$ & $<0.05$ \\
\hline
\end{tabular}

* Compared with the correspondent group injected saline into DRN + MRN

+ Compared with the correspondent group injected 5,7-DHT into DRN + MRN 


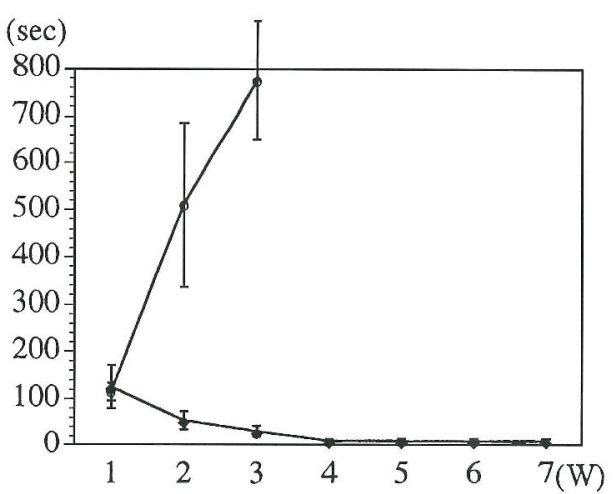

Fig. 2. Effect of raphe transplant of muricide. Muricide latency (mean \pm S.D) of raphe (open circles) and cerebellum (closed circles) transplanted groups at 1-7 weeks after transplantation.

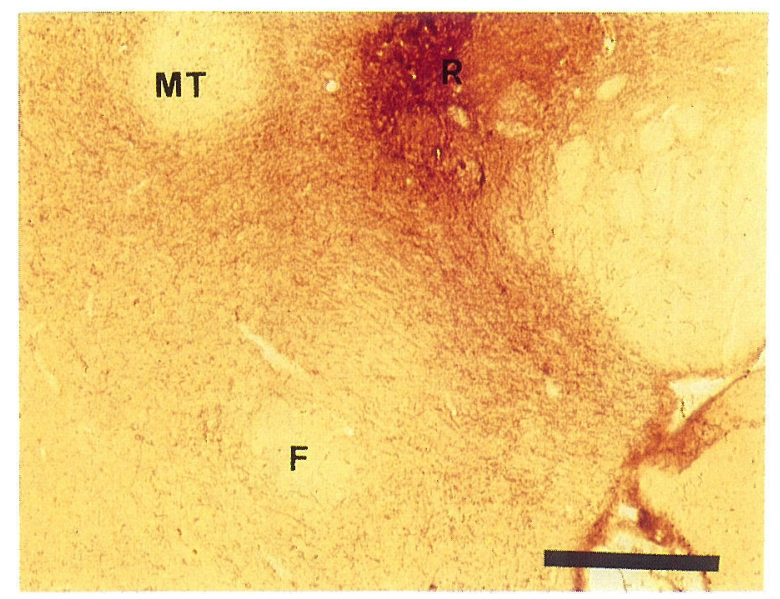

Fig. 3. Photomicrographs of serotonin-immunohistochemical stained section through the LHA of the raphe transplant rat. R, raphe tissue; F, fornix; MT, mammillothalamic tract. Bar $=$ $0.5 \mathrm{~mm}$.

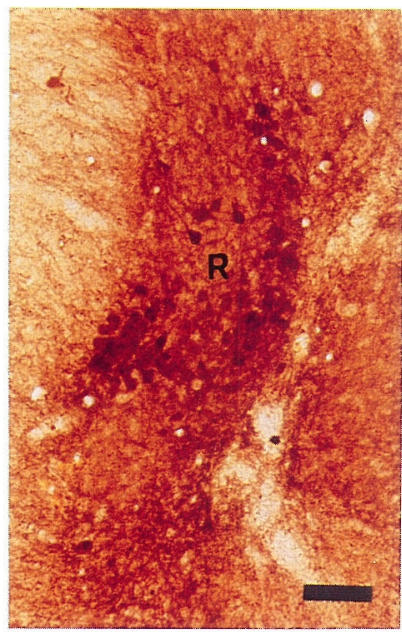

Fig. 4. Photomicrographs of serotonin-immunohistochemical stained section through the raphe transplant tissue. $B a r=200 \mu \mathrm{m}$.
Previous electrical stimulation studies have shown that the LHA is involved in aggressive behavior including muricide $[5,9,15]$. In the present study, the depression of serotonergic innervation in the LHA by 5,7-DHT and the reinnervation of serotonergic fibers in the LHA by raphe transplant directly demonstrate the serotonergic inhibitory role in the hypothalamic aggressive area.

By uncovering the neuronal mechanisms of aggression in experimental animals, including neuronal pathway, transmitters and receptors, we hope to elucidate the general mechanisms of this emotion. Although no one claims that the mechanisms are precisely the same in rats and humans, it seems clear from studies so far that there are similar pathways in rats and primates and possibly in humans, and several psychotropic drugs are useful for inhibiting aggression in rats, primates and humans. We are therefore confident that many of the findings in experimental animals will be applicable to humans. Because activating aggressive behavior, including violence, murder, and suicidal acts, involves dysfunctions of the brain's ability to control aggression, studies of the neural basis of aggressive behavior may help us further understand and treat these disturbances.

\section{References}

1. Blanchard, R. J. and Blanchard, D. C.: Aggressive behavior in the rat. Behav. Biol. 21; 197-224, 1997.

2. DiChiara, G., Camba, R. and Spano, P.F.: Evidence for inhibition by brain serotonin of mouse killing behaviour in rats. Nature 233; 272-273, 1971.

3. Grant, L. D., Cascina, D. V., Grossman, S. P. and Freedman, D. X.: Muricide after serotonin depleting lesions of midbrain raphé nuclei. Pharm. Biochemm. Behav. 1; 77-80, 1973.

4. Karli, P.: The Norway rats's killing response to the white mouse: an experimental analysis. Behaviour 10; 91-103, 1956.

5. Koolhaas, J. M.: Hypothalamically induced intraspecific aggressive behaviour in the rat. Exp. Brain Res. 32; 365-375, 1978.

6. Kulkarni, A. S., Rahwan, R. G. and Bocknik, S. E.: Muricidal block induced by 5-hydroxytryptophan in the rat. Arch. Int. Pharmacodyn. 201; 308-313, 1973.

7. Moyer, K.E.: Kinds of aggression and their physiological basis. Commun. Behav. Biol. A2; 65-87, 1968.

8. O'Boyle, M.: Rats and mice together: the predatory nature of the rat's mouse-killing response. Psychol. Bull. 82; 261-269, 1974.

9. Panksepp, J.: Aggression elicited by electrical stimulation of the hypothalamus in albino rats. Physiol. Behav. 6; 321-329, 1971.

10. Reis, D.: Central neurotransmitters in aggression. Res. Publ. Assoc. Res. Nerv. Mental Dis. 52; 119-148, 1974.

11. Sheard, M. H.: The effect of p-chiorophenylalanine on behavior in rats: relation to brain serotonin and 5-hydroxyindoleacetic acid. Brain Res. 15; 524-528, 1968.

12. Siegel, A. S. and Edinger, H.: Neural control of aggression and rage behavior. In "Handbook of the Hypothalamus Vol. 3", ed. by P. J. Morgane and J. Panksepp, Marchel Dekker, Inc., New York, 1981, pp. 203-240.

13. Waldbillig, R. J.: The role of the dorsal and medial raphe in the inhibition of muricide. Brain Res. 160; 341-346, 1979. 
14. Wasman, M. and Flynn, J. P.: Directed attack elicited from hypothalamus. Arch. Neurol. 6; 220-227, 1962.

15. Woodworth, C.H.: Attack elicited in rats by electrical stimulation of the lateral hypothalamus. Physiol. Behav. 6;
345-353, 1971.

16. Yamamoto, T. and Ueki, S.: Characteristics in aggressive behavior induced by midbrain raphe lesions in rats. Physiol. Behav. 19; 105-110, 1977. 